\title{
Schrödinger's phenotypes: herbarium specimens show two-dimensional images are both good and (not so) bad sources of morphological data
}

Authors Leonardo M. Borges ${ }^{1}$, Victor Candido Reis ${ }^{2}$, Rafael Izbicki ${ }^{2}$

${ }^{1}$ Departamento de Botânica, Universidade Federal de São Carlos, São Carlos, SP, Brazil

2 Departamento de Estatística, Universidade Federal de São Carlos, São Carlos, SP, Brazil

Correspondence Leonardo M. Borges

Departamento de Botânica, Universidade Federal de São Carlos, Rodovia Washington Luís, Km 235, São Carlos, SP, 13565-905, Brazil

aquitemcaqui@gmail.com

Author contribution LMB conceived the idea. LMB and RI designed the research. LMB collected original data and compiled legacy datasets. VCR and RI designed and performed the analyses. LMB and RI wrote the manuscript. All authors approved the final publication. LMB and RI contributed equally to this work.

Running headline Sourcing trait data from 2D specimen images 


\section{Schrödinger's phenotypes: herbarium specimens show two-dimensional images are both good and (not so) bad sources of morphological data}

Abstract 1. Museum specimens are the main source of information on organisms' morphological features. Although access to this information was commonly limited to researchers able to visit collections, it is now becoming freely available thanks to the digitization of museum specimens. With these images, we will be able to collectively build large-scale morphological datasets, but these will only be useful if the limits to this approach are well-known. To establish these limits, we used two-dimensional images of plant specimens to test the precision and accuracy of image-based data and analyses.

2. To test measurement precision and accuracy, we compared leaf measurements taken from specimens and images of the same specimens. Then we used legacy morphometric datasets to establish differences in the quality of datasets and multivariate analyses between specimens and images. To do so, we compared the multivariate space based on original legacy data to spaces built with datasets simulating image-based data.

3. We found that trait measurements made from images are as precise as those obtained directly from specimens, but as traits diminish in size, the accuracy drops as well. This decrease in accuracy, however, has a very low impact on dataset and analysis quality. The main problem with image-based datasets comes from missing observations due to image resolution or organ overlapping. Missing data lowers the accuracy of datasets and multivariate analyses. Although the effect is not strong, this decrease in accuracy suggests caution is needed when designing morphological research that will rely on digitized specimens.

4. As highlighted by images of plant specimens, 2D images are reliable measurement sources, even though resolution issues lower accuracy for small traits. At the same time, the impossibility of observing particular traits affects the quality of image-based datasets and, thus, of derived analyses. Despite these issues, gathering phenotypic data from two-dimensional images is valid and may support large-scale studies on the morphology and evolution of a wide diversity of organisms. 
Keywords digitization, morphology, trait, measurement, missing data, crowdsourcing

\section{Introduction}

Specimens held in natural history collections are our primary source of information on the diversity, morphological variety, and spatial and temporal distribution of living creatures. Based on this information, we obtain knowledge on many areas, such as environmental changes, public health, and evolution (Suarez and Tsutsui, 2004; Law and Salick, 2005; Babin-Fenske et al., 2008; Davis et al., 2015). Until recently, however, only people with access to museum collections could study these specimens. Now, almost all institutions are making their specimens more accessible through digitization.

Digitization methods vary as much as the biodiversity included in museums. Zoological collections may require the use of complex methods that better capture animal form, such as computer tomography, magnetic resonance imaging, or photogrammetry (e.g. Berquist et al., 2012; Falkingham, 2012; Keklikoglou et al., 2019). 2D imaging (photographs or flat scans), which has also been used for animals (e.g. Mantle et al., 2012; Schmidt et al., 2012), is particularly useful for flat specimens. Indeed, the flat, regular in size, and easy to image nature of plant samples has led to millions of plant specimens being available online (Le Bras et al., 2017; Soltis, 2017), and ready to be used as part of scientific research.

Taxonomy is one area in which digitization has been very fruitful. For example, automatic species identification software can substantially speed up taxonomy and specimen curation (Wang, Ji, Liang and Yuan, 2012; Remagnino et al., 2016; Carranza-Rojas et al., 2017). These tools, however, usually classify specimens by their overall image patterns, without making assumptions about organ identity (Wang, Ji, Liang and Yuan, 2012; Favret and Sieracki, 2016; Remagnino et al., 2016). Trait delimitation with images and derived data extraction (Corney, Tang, Clark, Hu and Jin, 2012; Corney, Clark, Tang and Wilkin, 2012; Wang, Lin, Ji and Liang, 2012; Gehan et al., 2017; Martineau et al., 2017), although promising, is still complex and underdeveloped. Even if we still have to wait for automatic data extraction to become highly efficient, imaging specimens facilitates data acquisition by researchers themselves, particularly if used in collective efforts to build comprehensive morphological datasets (e.g. O'Leary et al., 2013).

Such phenotypically diverse datasets may support advances in comparative and evolutionary biology (Laing et al., 2018). However, in comparison to genomic data, they take more time and money to produce (Burleigh et al., 2013). Tools that use specimens images are one option to overcome these challenges (Burleigh et al., 2013). However, this approach has limits. First, image resolution may limit observations, especially of small traits. Second, the nature of organisms themselves, or of specimen preparation, hides some features. For example, stamens in beans flowers are naturally hidden by the petals, and the wings of pinned butterfly specimens usually cover their legs. Although this is not a problem when one has access to specimens, it may become an issue for images. Thus, such variations in the preparation and nature of specimens may impact image-based data collection.

To evaluate the influence of these restrictions on the acquisition of morphological data from digitized specimens, we asked (1) if image measurements significantly differ from specimen measurements, 
and (2) if image-based datasets differ from the ones built with specimens to the point of affecting morphological analyses. To answer our first question, we compared measurements taken from herbarium specimens and their two-dimensional digital image. To answer our second question, we compared the results of multivariate analyses of plant specimens with analyses simulating image usage.

\section{Materials and Methods}

\subsection{A word on terminology}

Before outlining our analyses, we explain some of the terms we use, in particular accuracy and precision (for details, see Streiner and Norman, 2006). We consider precision to be an estimate of the variation between multiple measurements of the same feature. If values for these measurements do not differ widely, precision is high. Accuracy is treated as the difference between a measurement and a reference value and also applied here to judge the results of multivariate analyses. A close similarity between the measurement (or results) and the reference value indicates high accuracy. Because our goal is to evaluate the confidence of images as a data source in comparison to that obtained from specimens, we treated specimen data as the reference values against which we judged the accuracy of image data.

\subsection{Measurement precision and accuracy}

To test if measurements made from images and specimens differ, we measured different leaf parts of specimens belonging to five species (hereafter, measurement dataset). These species (Inga vera Willd., Ocotea divaricata Mez, Piper anisum (Spreng.) Angely, Smilax fluminensis Steud. and Sphagneticola trilobata (L.) Pruski) were selected to encompass different leaf morphologies, such as simple, compound, and lobed leaves with different base and apex shapes. Besides, by using these taxa, we avoided sampling size issues, as they are well represented in the Rio de Janeiro Botanical Garden herbarium (RB; acronym according to Thiers, 2020, continuously updated. See the supplementary material for information on measurements, sampling, and vouchers. Metric data is also available from MorphoBank project 3764 http://morphobank.org/permalink/?P3764). We used a digital caliper to measure specimens and the FSI Viewer v. 5.6.6 software (available from RB's website; http://jabot.jbrj.gov. br) to measure images of the same leaves from the same specimens. All measurements, both from images and specimens, were made twice to allow the following analyses.

First, we tested tested the precison of measurements made either from the specimens or from the images. To do so, we evaluated how the two repeated measurements are close to each other with the intraclass correlation coefficient (ICC) (Bartko, 1966). Then, we tested the hypothesis that images and specimens have the same average precision. For that, we compared the difference between the two repeated measurements made from images with those made from specimens with a paired t-test. The last test in this context evaluated if precision varies according to measurement scale. For that, we first divided the absolute difference between the two repeated measurements by their average to obtain mea- 
surements relative precision. Then, we plotted the relative precision of each observation against a scale reflecting overall trait size.

Having tested for precision, we computed the average of the two measurements of each variable. We used these averages to avoid bias in the following accuracy analyses. For each specimen and variable, we evaluated accuracy between measurements in the images to the specimens by testing their similarity with ICC (Bartko, 1966). We further evaluated accuracy testing the hypothesis that the median difference between image and specimen measurements is zero with a Wilcoxon signed-rank two-sample test (Wilcoxon, 1945). Finally, to quantify the relationship between measurement error and the magnitude of each variable, we plotted the relative error of the measurements (i.e., the absolute difference between image and specimen values divided by image mensuration) against the image measurement itself.

\subsection{Accuracy of image-based datasets}

To test if image-based data impacts analyses of morphological data, we compiled datasets of morphometric studies of different plant groups (Alcantara et al., 2013; Ames et al., 2008; Andres-Sanchez et al., 2009; Bello et al., 2018; Bünger et al., 2015; Egan, 2015; Kučera et al., 2006; Marhold, 1992; Poulsen and Nordal, 2005; Rose and Freudenstein, 2014; Slovák et al., 2012; Trovó et al., 2008). These datasets (hereafter original datasets) were used to generate three synthetic datasets, which were modified to include variation as if they had been compiled from images: the first included bias observed in the measurement accuracy analysis described above; the second included missing data; and the third included both measurement bias and missing data. We generated synthetic datasets with the following procedures.

First, we added noise to the original dataset using a zero-mean Gaussian distribution with varying values of variance. To do so, we modeled how the standard deviation of the measurements' error varies as a function of each variable's magnitude (See supplementary material). We then used this model to simulate different values of standard deviations for the Gaussian distribution of each variable, as to reproduce the same level of measurement error expected for that variable.

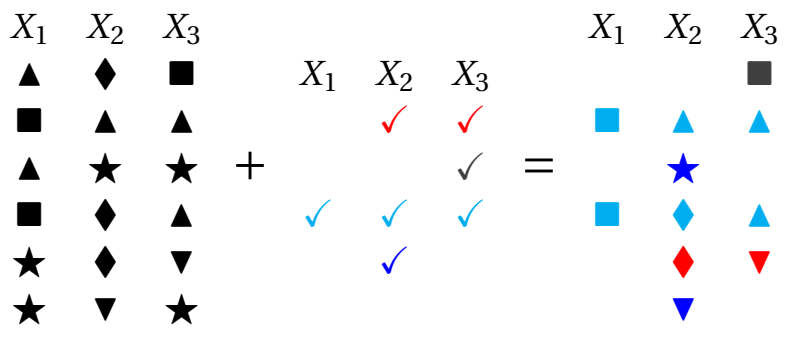

Figure 1: Illustration of the masking procedure. Left: original data set. Center: missing patterns obtained using digitized specimens; question marks indicate missing information and check-marks indicate available variables. Right: masked data used for constructing the synthetic dataset, obtained by applying a randomly chosen missing pattern (from the middle table) to each row of the original data set. 
Second, to simulate missing observations, we first checked which variables could be obtained from images on a set of up to ten digitized specimens from the same taxa of each morphometric study (see supplementary material for information on variables and vouchers). Using this information, we modeled patterns of missing data, which we used to mask observations in the original dataset, producing synthetic data including missing observations (Fig. 1). Finally, we combined the approaches just described to simulate the presence of both noise and missing data.

With original and synthetic datasets at hand, we compared agreement between results of Principal Component Analyses (PCA) of all of them. Besides being one of the most common methods in morphometric analyses, differences in PCA results summarize the overall variation of datasets being compared. Thus, PCA comparison captures differences between data acquisition methods (images vs. specimens).

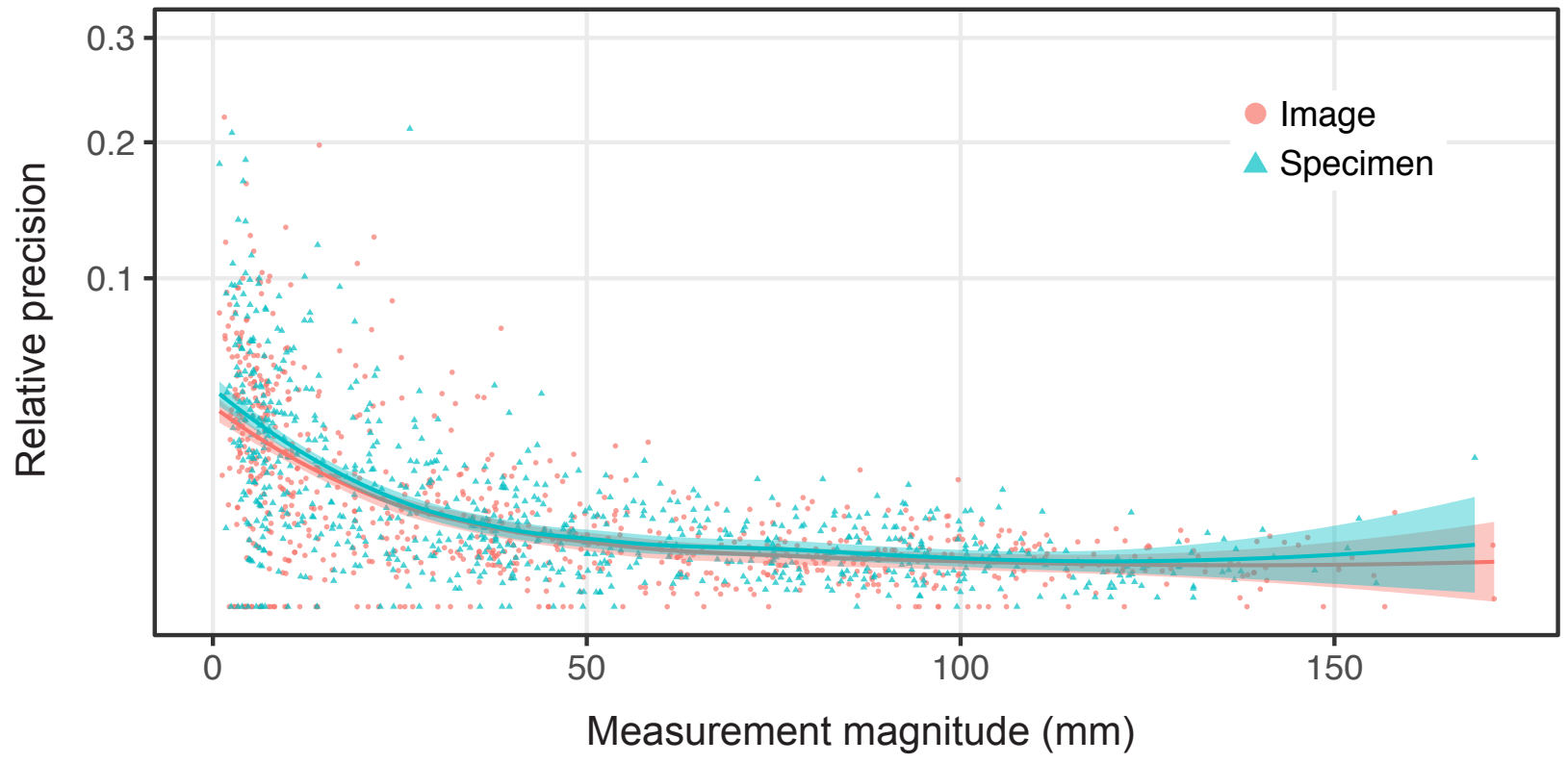

Figure 2: Relationship between the relative precision of measurements (i.e., the absolute difference between two repeated measurements divided by their average) and magnitude of each variable. Dots are different variable observations from the measurement dataset, either made on images (red) or on specimens (blue). The curves indicate the mean trend, along with their standard errors. $Y$-axis displayed in square root scale.

Our protocol first used pcaMethods (Stacklies et al., 2007) to run a Probabilistic PCA (Roweis, 1998) for each dataset, which automatically handles missing data with an expectation-maximization algorithm. We then measured the agreement between the first four principal components (PC) inferred for original and synthetic data by computing Pearson's correlation coefficient (Pearson, 1920) between the scores obtained for each observation. High correlation indicates agreement between analyses, and 
consequently between acquisition methods. However, as PC correlations may not capture overall dataset variation (Yang and Shahabi, 2004; Melo et al., 2015), we also used EvolQG (Melo et al., 2015) to measure PCA similarity (Yang and Shahabi, 2004) between original and synthetic data. PCA similarity weights PC correlation by their eigenvalues (Yang and Shahabi, 2004; Melo et al., 2015), and, thus, takes into account how much data variability is expressed in each PC.

The process described above was repeated 100 times (that is, 100 synthetic datasets were created), so we could evaluate the accuracy of image-based analyses to specimen-based PCAs with mean values and $95 \%$ confidence intervals.

\section{Results}

Measurements taken both from images and specimens have high internal precision, as seen in intraclass correlation coefficient values of $100.0 \%$. Moreover, the hypothesis that precision is the same between images and specimens was not rejected $(\mathrm{p}=0.678)$. Indeed, Fig. 2 indicates that measurements made on images and specimens have similar precision, and that, independently of the source, the precision decreases together with trait size.

Similarly, high ICC values indicate that measurements taken from images are accurate overall (Fig. 3). This lack of bias is reinforced by non-rejection of the hypothesis that they would not differ from specimens measurements (Fig. 4). At the same time, although measurements of larger traits are quite similar between specimens and images, measuring smaller organs with images is not as accurate. For example, blade length measurements of Ocotea divaricata or Piper anisum, which vary between 50$160 \mathrm{~mm}$, are extremely similar between specimens and images, as shown by the almost perfect fit of points to the identity line (curve $y=x$ ) in Fig. 3. On the other hand, points comparing petiole length measurements of the same species (2-15 mm long) are more dispersed around the identity line. This effect is clearly seen in the plot between relative error and specimen measurement values (Fig. 5), which shows that differences between image and specimen measurements are higher for smaller traits (below 30-40 mm).

All but two synthetic datasets (E2015, T2008) include missing data. The total proportion of missing observations, percentage of variables with at least $50 \%$ of observations, and variables that were impossible to observe from images (Table 1) vary between synthetic datasets, but at least one of these classes lacks more than $25 \%$ of observations for most datasets. Synthetic datasets also differ in proportions of missing data by type of variable (continuous, categorical, discrete, or ratio; Table 2), with continuous variables being more commonly absent. Even though patterns of missing data may differ between datasets, correlation coefficients indicate that drops in accuracy are more related to absences spread in the dataset than to complete lack of a particular variable (Table 3).

The analyses of original and synthetic datasets (Fig. 6) show that the results of principal component analyses based on images are affected by noise and missing data. Nonetheless, noise effect is almost non-existent, as shown by values near $100 \%$ for both PCA similarity and PC correlation for the 

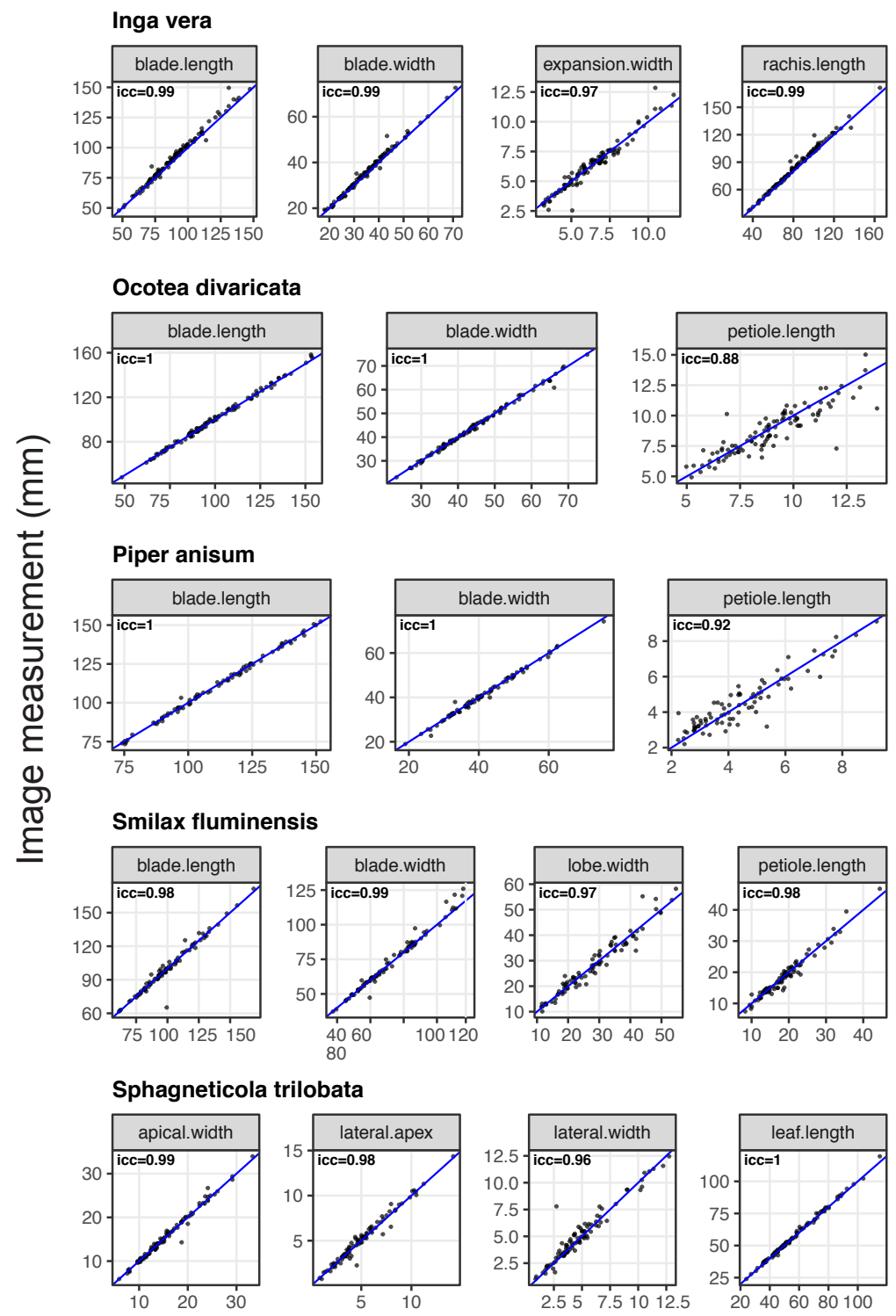

\section{Specimen measurement $(\mathrm{mm})$}

Figure 3: Scatter plots with measurements made from specimen versus measurements made from the images on the measurement dataset. Blue lines indicate the line $y=x$, in which both measurements are the same. Each panel also shows the Pearson's correlation coefficient between the measurements. 
first and second principal components. On the other hand, accuracy drops in face of missing data, and particularly due to the joint effect of noise and missing data.

Table 1: Proportion of missing data in each dataset according to three criteria: total percentage of missing data (total); percentage of variables with at least $50 \%$ of missing observations (at least $50 \%$ ); percentage of variables impossible to score from images (100\%).

\begin{tabular}{llll}
\hline Dataset & total \% & at least 50\% & $100 \%$ \\
\hline A2013 & $50 \%$ & $56.25 \%$ & $0 \%$ \\
A2008 & $44.39 \%$ & $45.12 \%$ & $19.51 \%$ \\
A2009 & $69.17 \%$ & $100 \%$ & $8.33 \%$ \\
B2018 & $37.5 \%$ & $35.71 \%$ & $35.71 \%$ \\
B2015 & $19.44 \%$ & $8.33 \%$ & $0 \%$ \\
E2015 & $0 \%$ & $0 \%$ & $0 \%$ \\
K2006i & $30.72 \%$ & $41.18 \%$ & $5.88 \%$ \\
K2006p & $30.72 \%$ & $41.18 \%$ & $5.88 \%$ \\
M1992 & $10 \%$ & $10 \%$ & $10 \%$ \\
P2005 & $42.97 \%$ & $43.75 \%$ & $12.5 \%$ \\
R2014 & $60.61 \%$ & $63.64 \%$ & $45.45 \%$ \\
S2012 & $43.56 \%$ & $44 \%$ & $24 \%$ \\
T2008 & $0 \%$ & $0 \%$ & $0 \%$ \\
\hline
\end{tabular}

Table 2: Percentage of missing data by type of variable for each data set used to build the masks with missing data pattern.

\begin{tabular}{lllll}
\hline Dataset & Continuous (mm) & Categorical & Discrete & Ratio \\
\hline A2013 & $50 \%$ & - & - & - \\
A2008 & $39.39 \%$ & $53.33 \%$ & $52.73 \%$ & $37.65 \%$ \\
A2009 & $70 \%$ & - & $60 \%$ & - \\
B2018 & $50 \%$ & $16.67 \%$ & $0 \%$ & - \\
B2015 & $19.44 \%$ & - & - & - \\
E2015 & $0 \%$ & - & - & - \\
K2006i & $46.67 \%$ & - & $13.89 \%$ & $0 \%$ \\
K2006p & $46.67 \%$ & - & $13.89 \%$ & $0 \%$ \\
M1992 & $20 \%$ & $0 \%$ & $0 \%$ & - \\
P2005 & $32.81 \%$ & $56.25 \%$ & $43.75 \%$ & - \\
R2014 & $51.04 \%$ & - & $58.33 \%$ & $100 \%$ \\
S2012 & $41.11 \%$ & $56.94 \%$ & $31.75 \%$ & - \\
T2008 & $0 \%$ & - & $0 \%$ & - \\
\hline
\end{tabular}

\section{Discussion}

Here we used a set of leaf measurements and legacy morphometric studies to evaluate the precision and accuracy of morphological data gathered from images of herbarium specimens. We found image measurements to be highly precise and accurate, even though there is a small drop in accuracy for smaller 
Table 3: Spearman correlation coefficients and their respective p-values for comparing the relationship between the accuracy in the estimation of each principal component/the PCA similarity (according to the "M" scheme) and the percentage of missing observations. total\% - total percentage of missing data in the dataset; at least $50 \%$ - variables with at least $50 \%$ of missing observations; $100 \%$ - variables impossible to score from images.

\begin{tabular}{llll}
\hline PCA Component & total $\%$ & at least $50 \%$ & $100 \%$ \\
\hline 1 & $-0.45(0.12)$ & $-0.48(0.10)$ & $-0.15(0.63)$ \\
2 & $-0.78(<0.01)$ & $-0.79(<0.01)$ & $-0.51(0.08)$ \\
3 & $-0.65(0.02)$ & $-0.66(0.01)$ & $-0.41(0.16)$ \\
4 & $-0.67(0.01)$ & $-0.68(0.01)$ & $-0.30(0.32)$ \\
PCA Sim & $-0.73(<0.01)$ & $-0.73(<0.01)$ & $-0.78(<0.01)$ \\
\hline
\end{tabular}

traits. On the other hand, missing observations may decrease the accuracy of image-based morphological datasets. Below we discuss the problems, advantages, and consequences of collecting morphological data from digitized specimens.

\subsection{Measurement precision and accuracy}

Measurements taken from digitized herbarium specimens are as precise as the ones made on actual specimens. In fact, image measurements are in the confidence limits of being more precise (Fig. 2). An increase in precision has been seen for other imaging systems, as micro-computed tomography (Simon and Marroig, 2015). Thus, as we have focused only on leaves of particular species, it is possible analyses including a wider diversity of morphological variables to find the same for images of herbarium specimens.

Image measurements are also accurate, as indicated by their high similarity to specimen measurements (Fig. 3) and overall lack of bias (Fig. 4). Agreement between data gathered from images and specimens was also seen for the extraction of morphological features using artificial intelligence, which compared measurements taken by computers and researchers (Corney, Clark, Tang and Wilkin, 2012). Similar results for different organisms and imaging methods (Simon and Marroig, 2015; Aldridge et al., 2005) reinforce that high-confidence measurements can be obtained from digitized specimens. Nonetheless, we have also evidenced particular issues in the accuracy of image-based measurements and overall data acquisition.

\subsection{Noise}

The first problem with image use is a drop in measurement accuracy as variables scale down (Fig. 4). Measurements of smaller features, particularly those below 30-40 mm (Fig. 5), differ more between images and specimens than do larger structures. This association between accuracy error and scale was to be expected, as measurement precision lowers for smaller traits (Fig. 2). Such a bias in precision is not limited to images and is seen in our specimen measurements (Fig. 2), as well as for bird bones (Yezerinac et al., 1992) and Drosera (Droseraceae) leaves (Hoyo and Tsuyuzaki, 2013), for example. In this con- 

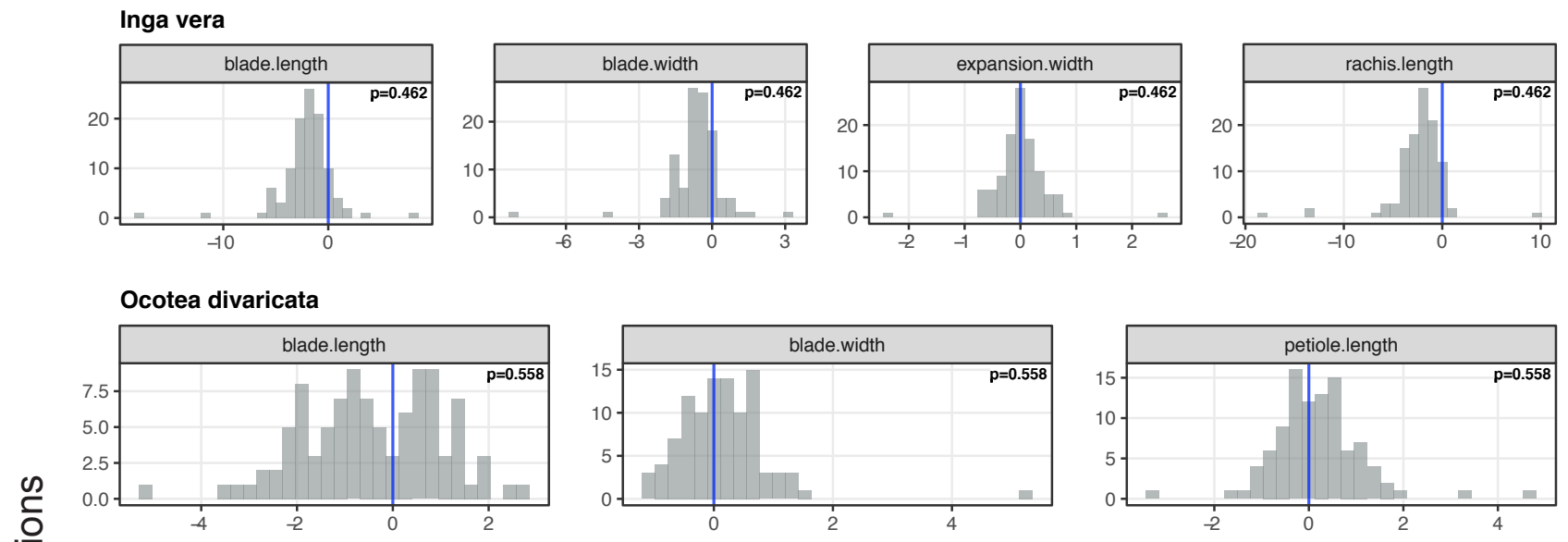

\section{Piper anisum}
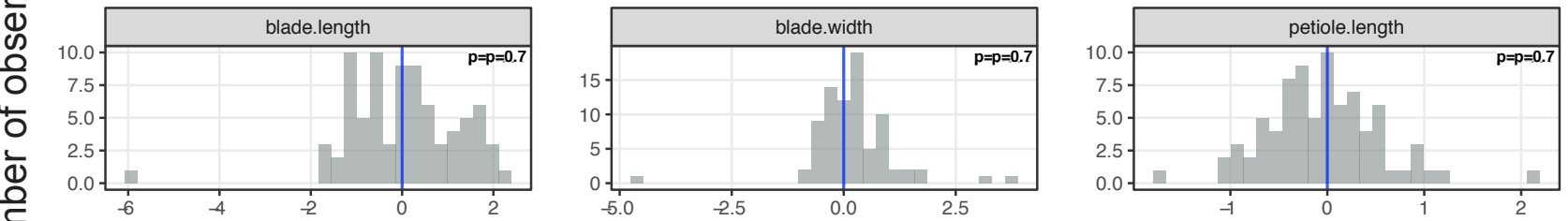

\section{Smilax fluminensis}
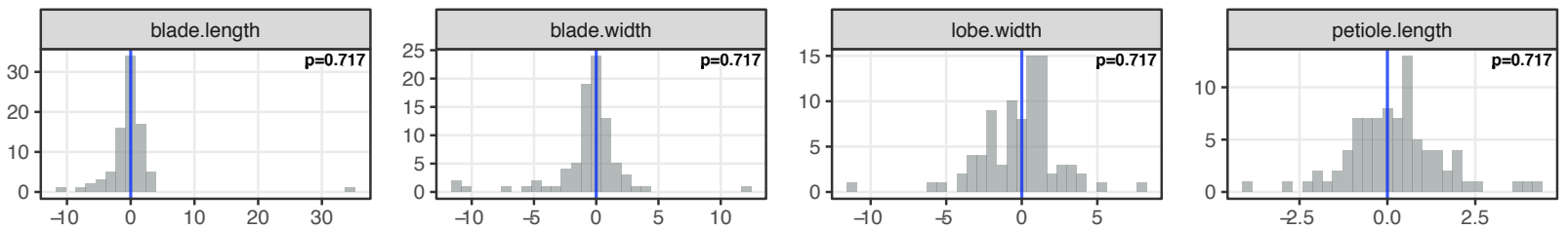

\section{Sphagneticola trilobata}
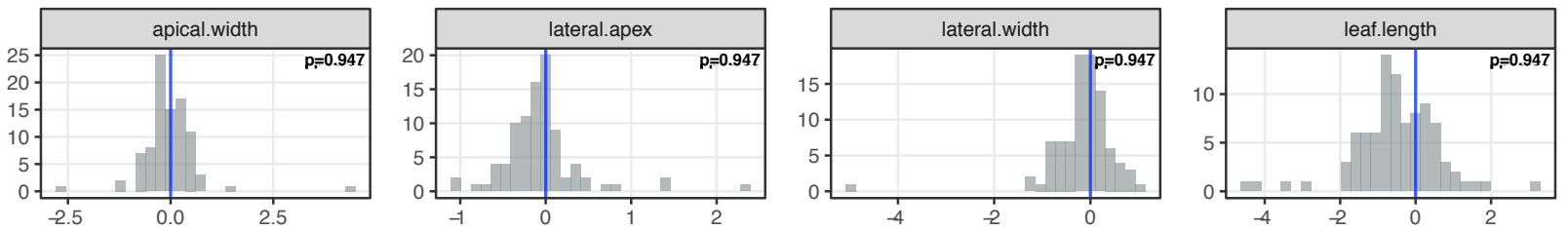

\section{Difference between specimen and image measurements $(\mathrm{mm})$}

Figure 4: Histogram of the difference between measurements made in the specimens and images on the measurement dataset. Vertical lines indicate the line $x=0$, in which the difference between the measurements is zero. Each panel also shows the p-values for testing the hypothesis that the median difference between image and specimen measurements is zero. 
text, our results do not mean images are worse measurement sources. However, while we can increase precision and accuracy by studying specimens with different magnification tools, zooming images is restricted by resolution. Image resolution may not be a problem for high quality images (e.g. JStor Global Plants; https : / plants . jstor . org/), but as digitization methods vary (Tulig et al., 2012; Takano et al., 2019; Sweeney et al., 2018), databases will differ in how they are prone to measurement errors.

Even though measurement errors will always be present, their effect is not strong. Our results show that datasets compiled from images and specimens are essentially the same (Fig. 6), especially as indicated by high PCA similarity (Yang and Shahabi, 2004; Melo et al., 2015). It is possible our results are biased, as most variables in our legacy datasets fall outside the range of sizes we found to be problematic for plant specimens (below 30-40 mm). Thus, researchers working on smaller organs or with small organisms should bear this potential bias in mind. Nonetheless, as seen for other taxa and methods using image-based measurements (e.g. Bruner, Emiliano and Costantini, David and Fanfani, Alberto and Dell'Omo, Giacomo, 2005; Corney, Tang, Clark, Hu and Jin, 2012; Corney, Clark, Tang and Wilkin, 2012; Chang and Alfaro, 2016), specimens two-dimensional images are good sources of metric data. Of course, this will only hold for traits that can be observed in the images.

\subsection{Missing data}

Another problem in our synthetic databases was the pervasive presence of missing data (Table 1), particularly of continuous variables (Table 2). As discussed above, these absences are likely due to image resolution issues, which hamper the observation of small features. Nonetheless, the extent of this problem is particular to each dataset. For example, the only two datasets with no missing data (T2008 and E2015; 1) also include variables that are smaller than $30 \mathrm{~mm}$. At the same time, larger organs that were concealed during specimen preparation or due to their overlapping nature (e.g. petals usually hide both androecium and gynoecium) can not be observed and, thus, bias data collection. Feature-related bias is also seen in automatic trait recognition in which organ identity affects accuracy (Younis et al., 2018). Similar examples come from our datasets. If Trovó et al., 2008 had investigated the morphology of flowers (usually smaller than $2 \mathrm{~mm}$ in Eriocaulaceae), it would not be possible to reproduce their data solely with images. This is the case with the T2013 dataset (Alcantara et al., 2013), in which the main source of missing data are organs concealed within the Bignoniaceae tubular flowers. Even though we can manipulate specimens to expose such hidden features, the same can not be done with herbarium images.

Indeed, missing data lower PC correlation and PCA similarity (Fig. 6). This trend is reinforced by the negative correlation between the proportion of missing data in synthetic dataset and PCA similarity or PC estimation (Table 3). Accuracy drops are stronger for the second, and especially for the third and fourth PCs. Although poor estimation of third and fourth PCs may not be a big problem, inaccuracy in estimation of the first two components and overall multivariate space, expressed in PCA similarity, points to significant differences between original and synthetic datasets. These differences could bias biological interpretations (Yezerinac et al., 1992) of studies relying on acquisition of morphological data, such as phenotypic evolution, disparity, modularity, phylogenetic inference, and others (e.g. Wiens, 
2003; Zaragüeta-Bagils and Bourdon, 2007; Catalano et al., 2010; Klingenberg and Gidaszewski, 2010; Vasconcelos et al., 2018; Castiglione et al., 2019; Dellinger et al., 2019; Gallaher et al., 2019; Guillerme and Cooper, 2018).

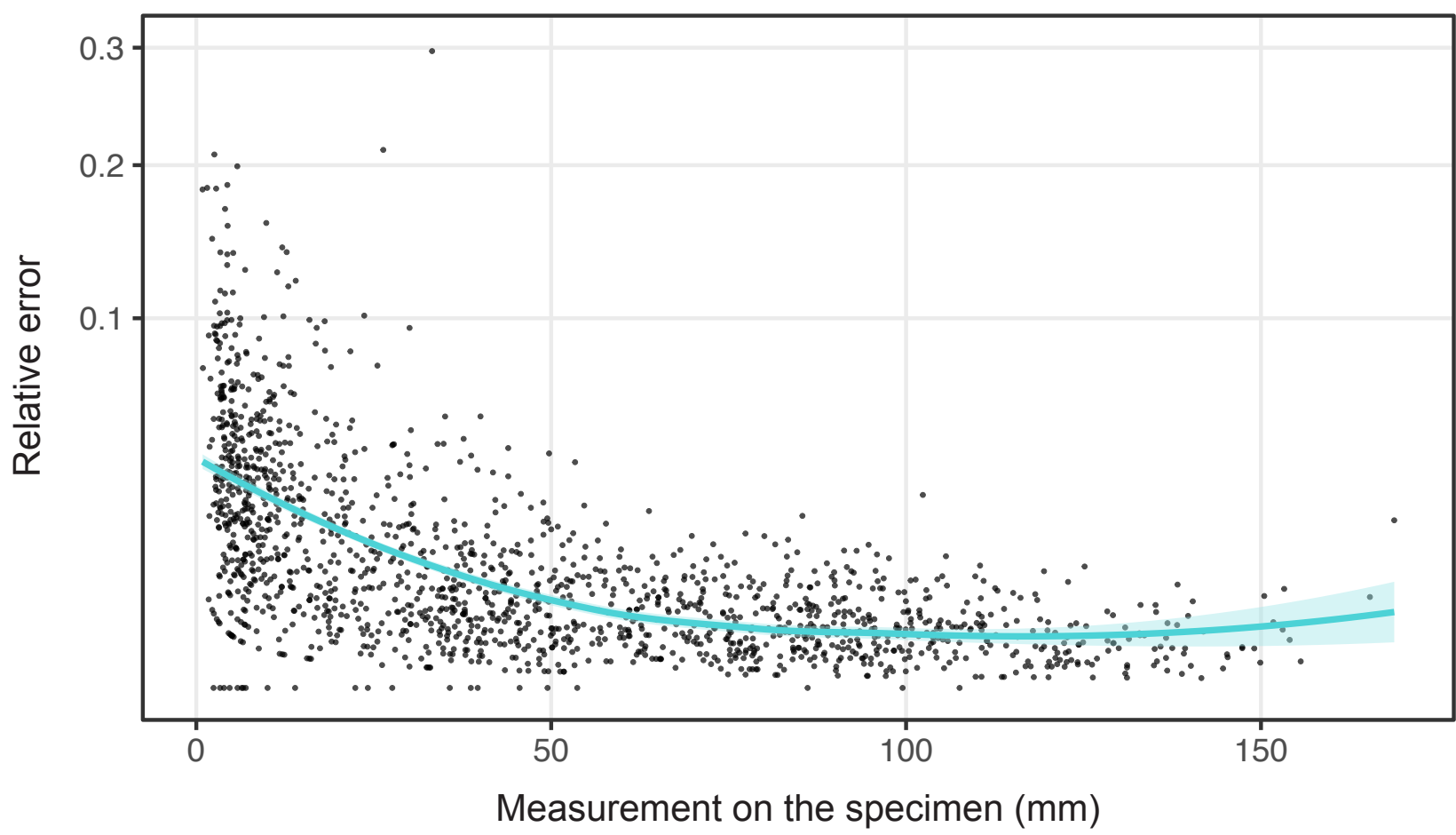

Figure 5: Relationship between the relative error of the measurements (i.e., the absolute difference between image and specimen measurements divided by specimen measurement) and the magnitude of each variable. Each dot represents a different observation from a variable in the measurement dataset. The curve indicates the mean trend, along with its standard error. $Y$-axis is displayed in a square root scale.

\subsection{Joint effect of measurement noise and missing data}

Above, we analyzed the individual effects of measurement noise and missing data over dataset accuracy. However, most real datasets likely will include both issues. Our results indicate they interact, further dropping PC correlation and PCA similarity (Fig. 6). For a few cases in which noise is stronger than missing data (e.g. third and/or fourth PCs in B2018, and M1991), the joint effect improves component estimation, likely by removing noisy variables. Apart from those exceptions, the drop in accuracy when both noise and missing data are present may affect biological interpretations (see above). Particularly, rates of false positive and false negative conclusions may increase for study systems with low internal variation, which is common in comparisons of closely related taxa (Yezerinac et al., 1992). 


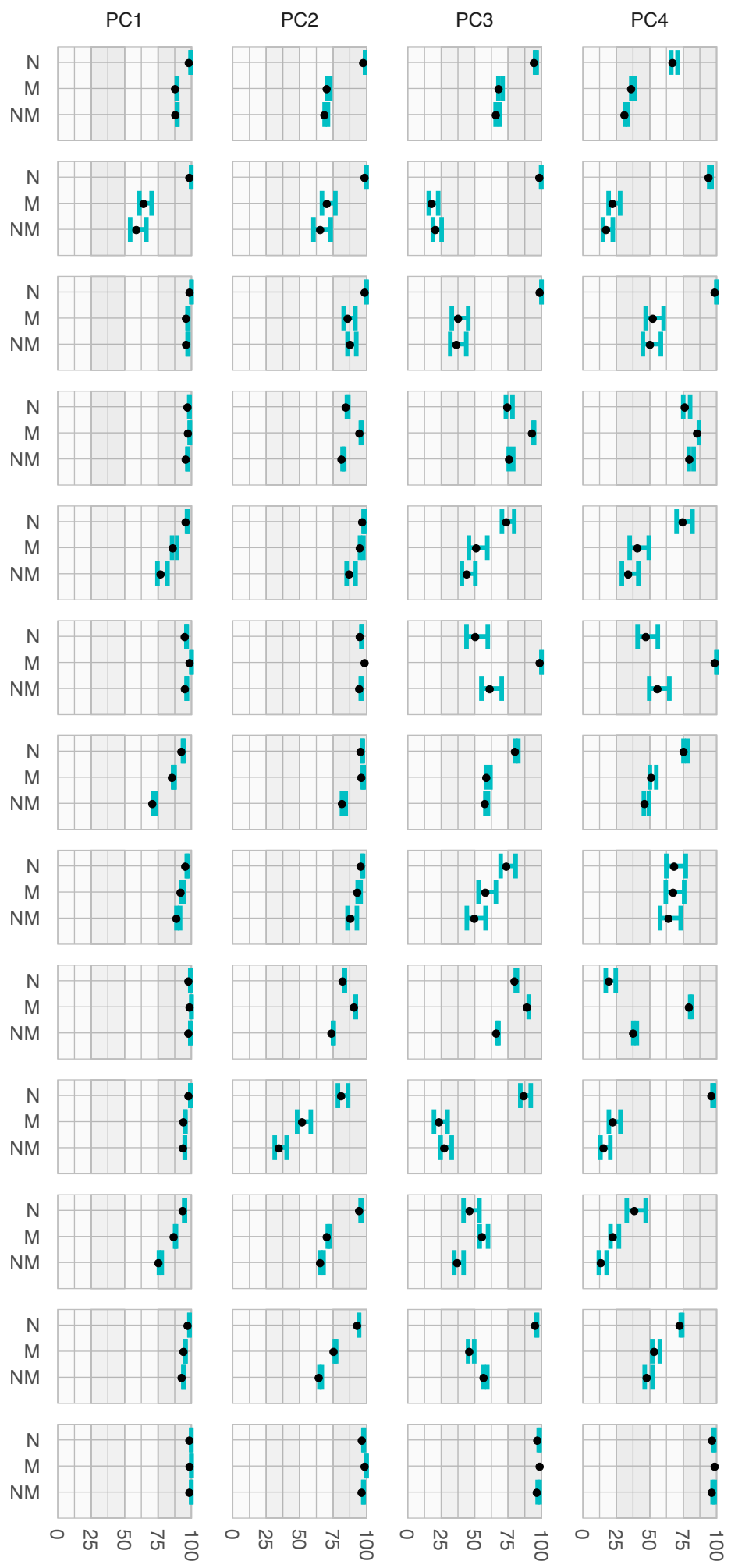

Average absolute correlation (\%)
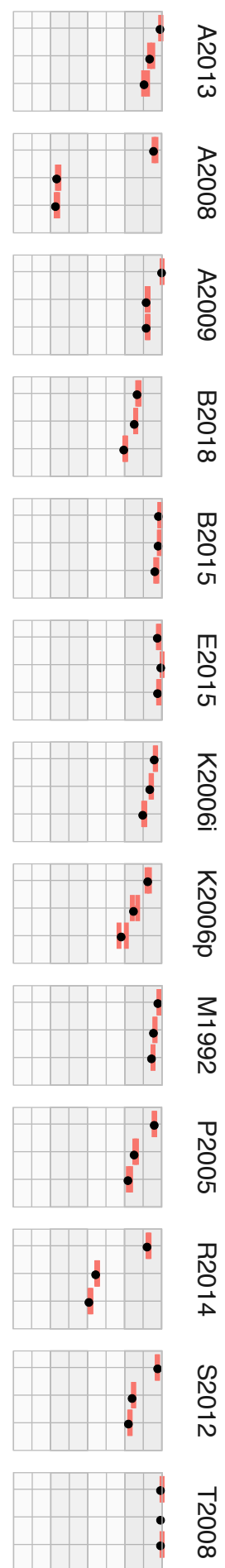

○ ํํㅇ जै $\overrightarrow{8}$

PCA similarity $(\%)$

Figure 6: (Left) Accuracy (and 95\% confidence intervals) in estimating the first four principal components using images (synthetic datasets). (Right)3PCA Similarity between synthetic datasets versus original. $100 \%$ values equal maximum accuracy for both analyses. $\mathrm{N}$ - synthetic dataset with noise on the same level as those observed in Figure 3. M - synthetic dataset without variables that can not be acquired from images. NM - synthetic dataset with both noise and missing data. 
Although the joint effect of noise and missing data may be serious, its influence will vary as datasets differ in the amount of missing data and presence of noise, which are linked to traits of interest size, image resolution, and organ overlap. Hence, before relying solely on morphological data compiled from digitized specimens, researchers should first test how image use affects their particular studies. For some cases, the overall accuracy we found in our analyses would be problematic (Fig. 6). For others, this accuracy would be just fine.

\subsection{Consequences for image-based morphological data}

When compiling morphological data from images of herbarium specimens, measurement noise is not a problem, particularly if variables are outside the size range affected by resolution. Hence, even though resolution may vary among digitization methods (Tulig et al., 2012; Takano et al., 2019; Sweeney et al., 2018), measuring them instead of specimens is an effective, less expensive (no need for traveling or loans), and, more important, reliable method for data acquisition.

On the other hand, missing data has a higher impact on data collection. This impact, however, will vary between different groups, particular morphologies, and specific research questions. For the cases in which missing data is relevant, researchers can improve data quality with particular strategies, used individually or in combination: 1 ) complete data with imputation methods; 2) exclude problematic variables or observations; 3 ) use specimens to fill gaps.

Data imputation methods estimate missing values in a dataset based on a variable's internal variance, or on its associations to other variables in the dataset (Frane, 1976; Rubin, 1976; Little and Rubin, 2019). Here, for example, our PCA analyses used a relatively simple imputation method (Roweis, 1998). Although not free of issues (Little and Rubin, 2019), data estimation methods are powerful tools to improve results without compromising biological meaning (König et al., 2019).

Data imputation, however, will be inaccurate in cases where almost all observations of a given variable are missing. In this situation, it is sometimes better to exclude the variables from the analysis, particularly if it is highly correlated to other variables (Hemel et al., 1987). This way, variable exclusion is less likely to impact analyses or at least will have lower impact, as seen for the smaller negative correlation of fully absent variables to PC estimation and PCA similarity (Table 3). On the other hand, depending on the reason why a feature is missing, variable exclusion may bias results (Little and Rubin, 2019). Thus, it is important to understand the performance of data imputation and data exclusion when dealing with missing observations (Musil et al., 2002; Saunders et al., 2006; Horton and Kleinman, 2007; Enders, 2008).

If variable exclusion and data imputation are not feasible for a given case, such as a complete lack of observations on an important variable, researchers can use specimens to fill gaps in the dataset. Although not as practical as the other options, this approach can at least speed up work inside museums. When access to collections is limited, it is possible to combine examination of a minimum number of specimens and data imputation. These options exemplify how there is no single solution to circumvent missing data issues.

A more powerful approach to limit missing data - and further lower measurement noise - is to ex- 
pand digital collections to include images with different magnifications, images exposing structures commonly hidden, or images taken from multiple angles. Increasing magnification and exposing hidden traits are complex and time-consuming but could be achieved if researchers fed back to institutions the images obtained while studying the specimens. Multi-camera imaging is particularly helpful for zoological specimens (Tegelberg et al., 2014; Price et al., 2018; Hereld et al., 2017; Ströbel et al., 2018; Hereld and Ferrier, 2019), as it allows proper visualization of traits that are hidden or distorted on images in a single view. Combining multi-view imaging and production lines (Tegelberg et al., 2014) is one efficient option to generate imagery that supports both 3D reconstructions (Hereld et al., 2017; Ströbel et al., 2018; Hereld and Ferrier, 2019) and accurate morphological data acquisition from the original two-dimensional images themselves, as shown here.

Despite particularities discussed above, digitization opened new frontiers for image-based phenotyping. Up to now, the bulk of research using specimen images focused on species identification (e.g. MacLeod, 2007; Cope et al., 2012; Wang, Lin, Ji and Liang, 2012; Wang, Ji, Liang and Yuan, 2012; MacLeod and Steart, 2015; Unger et al., 2016; Remagnino et al., 2016; Carranza-Rojas et al., 2017), or on shape analysis of particular organs (e.g. Corney, Clark, Tang and Wilkin, 2012; Smith and Kriebel, 2018; Reginato and Michelangeli, 2016). For studies focused on individual organs, datasets usually included only images of the focal traits. Now, the millions of images of herbarium specimens available make a wider diversity of phenotypes freely accessible, an accomplishment soon to be followed by zoological collections. In turn, easy access to specimen images supports data collection with crowdsourcing, a relatively fast and cheap way to obtain high-quality data (Chang and Alfaro, 2016; Zhou et al., 2018; O'Leary et al., 2018). Moreover, crowdsourced datasets may also aid the improvement of automatic extraction methods (Burleigh et al., 2013; Zhou et al., 2018), or be used in parallel with them to improve data collection. For example, while machines still can not differentiate overlapped plant organs (Gehan and Kellogg, 2017), an automatic approach can be complemented by human input, as we can easily identify and overcome overlapping issues. Independently of particular data collection strategies and of the need to be careful with missing observations, we reinforced here that digitized specimens are good sources of morphological data.

\subsection{A note on data sharing}

One of the main advantages of collecting data from digitized specimens is their free availability in digital repositories. It is only fair that sharing extends to data as well. However, at least for plant morphometric studies, this is not frequently done. For example, our original dataset came from just a few authors, who shared raw observations at publication time or kindly replied to our data requests. A much larger number of studies, however, presented only tables with mean and standard deviation values, which do not allow data reuse or verification.

If we aim to build comprehensive datasets, we have to shift this trend and increase the use of tools such as MorphoBank (https : //morphobank .org; O'Leary and Kaufman, 2011) to share morphological data. Data sharing improves scientific work, and reduces redundant data collection, particularly if data 
is strongly integrated with ontologies (Deans et al., 2015) and linked to vouchers and scientific names in curated databases (Bruneau et al., 2019).

\section{Conclusion}

Measuring digitized specimens is a reliable data collection method, as demonstrated by a high correlation between measurement from specimens and images, even though resolution introduces measurement errors on small scales. At the same time, as it is impossible to manipulate images, particular features can not be observed and will be missing from image-based datasets. Even though there are some options to reduce the effects of missing data on analyses, the impact of this more serious problem has to be evaluated for each case.

Overall, we showed that accurate morphological data can be sourced from two-dimensional specimen images. For now, the millions of digital specimens (plus data on their distribution, ecology, and phenology) already available for plants (Le Bras et al., 2017; Soltis, 2017; Willis et al., 2017) put Botany at the front line of collective morphological data acquisition. As digitization expands to other groups, we will be able to merge big-data on geographical distribution, genomes, and morphology to unveil new knowledge on patterns and processes of phenotypic diversity and evolution.

\section{Acknowledgments}

We are grateful to Abubakar Bello, Axel Poulsen and Inger Nordal, David Spooner and Mercedes Ames, Jeffrey Rose, Karol Marhold, Marcelo Trovó, Marek Slovák, Mariana Bünger, M. Montserrat MartínezOrtega, Suzana Alcantara, and Vanessa Staggemeier for sharing their datasets, either at publication time or directly to us. We also thank Sara Mortara for initial help on research design and data collection, Kaori Nagata and Jon Wilcox for linguistic editing, as well as Rafaela C. Forzza, Paula Leitman, Fabiana L. R. Filard, and the RB staff for support during work at the herbarium. Anna Penna, Diogo Melo, Matheus F. Santos, and Monique N. Simon's bright comments and suggestions helped us improve early versions of this work. Finally, we thank the editors and reviewers for their thoughtful suggestions. RI was supported in part by FAPESP (2019/11321-9) and CNPq (306943/2017-4). The authors declare no conflict of interest.

\section{Data accessibility statement}

All data and code used here are available from GitHub (DOI: 10.5281/zenodo.3924506). Leaf measurements are also individually available from MorphoBank (project 3764 http : / morphobank . org/permalink/ ?P3764). 


\section{References}

Alcantara, S., de Oliveira, F. and Lohmann, L. (2013), 'Phenotypic integration in flowers of neotropical lianas: diversification of form with stasis of underlying patterns', Journal of Evolutionary Biology 26(10), 2283-2296. DOI: 10.1111/jeb.12228. 4, 11

Aldridge, K., Boyadjiev, S. A., Capone, G. T., DeLeon, V. B. and Richtsmeier, J. T. (2005), 'Precision and error of three-dimensional phenotypic measures acquired from 3dMD photogrammetric images', American Journal of Medical Genetics Part A 138(3), 247-253. DOI: 10.1002/ajmg.a.30959. 9

Ames, M., Salas, A. and Spooner, D. M. (2008), 'A morphometric study of species boundaries of the wild potato Solanum series Piurana (Solanaceae) and putatively related species from seven other series in Solanum sect. Petota', Systematic Botany 33(3), 566-578. DOI: 10.1600/036364408785679789. 4

Andres-Sanchez, S., Rico, E., Herrero, A., Santos-Vicente, M. and MartINez-Ortega, M. M. (2009), 'Combining traditional morphometrics and molecular markers in cryptic taxa: towards an updated integrative taxonomic treatment for Veronica subgenus Pentasepalae (Plantaginaceae sensu APG II) in the western Mediterranean', Botanical Journal of the Linnean Society 159(1), 68-87. DOI: 10.1111/j.10958339.2008.00917.x. 4

Babin-Fenske, J., Anand, M. and Alarie, Y. (2008), 'Rapid morphological change in stream beetle museum specimens correlates with climate change', Ecological Entomology 33(5), 646-651. DOI: 10.1111/j.1365-2311.2008.01018.x. 2

Bartko, J. J. (1966), 'The intraclass correlation coefficient as a measure of reliability', Psychological reports 19(1), 3-11. DOI: 10.2466/pr0.1966.19.1.3. 3, 4

Bello, A., Stirton, C., Chimphango, S. and Muasya, A. (2018), 'Morphological evidence for introgressive hybridization in the genus Psoralea L. (Psoraleeae, Fabaceae)', South African Journal of Botany 118, 321-328. DOI: 10.1016/j.sajb.2018.02.407. 4

Berquist, R. M., Gledhill, K. M., Peterson, M. W., Doan, A. H., Baxter, G. T., Yopak, K. E., Kang, N., Walker, H., Hastings, P. A. and Frank, L. R. (2012), 'The Digital Fish Library: using MRI to digitize, database, and document the morphological diversity of fish', PLoS One 7(4). DOI: 10.1371\%2Fjournal.pone.0034499. 2

Bruneau, A., Borges, L. M., Allkin, R., Egan, A. N., Estrella, M. d. l., Javadi, F., Klitgård, B., Miller, J. T., Murphy, D. J., Sinou, C., Vatanparast, M. and Zhang, R. (2019), 'Towards a new online species-information system for legumes', Australian Systematic Botany 32(6), 495-518. DOI: 10.1071/sb19025. 16

Bruner, Emiliano and Costantini, David and Fanfani, Alberto and Dell'Omo, Giacomo (2005), 'Morphological variation and sexual dimorphism of the cephalic scales in Lacerta bilineata', Acta zoologica 86(4), 245-254. DOI: 10.1111/j.1463-6395.2005.00206.x. 11 
Bünger, M. d. O., Einsehlor, P., Figueiredo, M. L. N. and Stehmann, J. R. (2015), 'Resolving Species Delimitations in the Eugenia involucrata Group (Eugenia sect. Phyllocalyx - Myrtaceae) with Morphometric Analysis', Systematic Botany 40(4), 995-1002. DOI: 10.1600/036364415x690030. 4

Burleigh, J. G., Alphonse, K., Alverson, A. J., Bik, H. M., Blank, C., Cirranello, A. L., Cui, H., Daly, M., Dietterich, T. G., Gasparich, G. et al. (2013), 'Next-generation phenomics for the Tree of Life', PLoS Currents 5. DOI: 10.1371/currents.tol.085c713acafc8711b2ff7010a4b03733. 2, 15

Carranza-Rojas, J., Goeau, H., Bonnet, P., Mata-Montero, E. and Joly, A. (2017), 'Going deeper in the automated identification of Herbarium specimens', BMC Evolutionary Biology 17(1), 181. DOI: 10.1186/s12862-017-1014-z. 2, 15

Castiglione, S., Serio, C., Tamagnini, D., Melchionna, M., Mondanaro, A., Di Febbraro, M., Profico, A., Piras, P., Barattolo, F. and Raia, P. (2019), 'A new, fast method to search for morphological convergence with shape data', PloS one 14(12). DOI: 10.1371/journal.pone.0226949. 12

Catalano, S. A., Goloboff, P. A. and Giannini, N. P. (2010), 'Phylogenetic morphometrics (I): the use of landmark data in a phylogenetic framework', Cladistics 26(5), 539-549. DOI: 10.1111/j.10960031.2010.00302.x. 12

Chang, J. and Alfaro, M. E. (2016), 'Crowdsourced geometric morphometrics enable rapid large-scale collection and analysis of phenotypic data', Methods in Ecology and Evolution 7(4), 472--482. DOI: 10.1111/2041-210X.12508. 11, 15

Cope, J. S., Corney, D., Clark, J. Y., Remagnino, P. and Wilkin, P. (2012), 'Plant species identification using digital morphometrics: A review', Expert Systems with Applications 39(8), 7562-7573. DOI: 10.1016/j.eswa.2012.01.073. 15

Corney, D., Clark, J. Y., Tang, H. L. and Wilkin, P. (2012), 'Automatic extraction of leaf characters from herbarium specimens', Taxon 61 (1), 231-244. DOI: 10.1002/tax.611016. 2, 9, 11, 15

Corney, D. P., Tang, H. L., Clark, J. Y., Hu, Y. and Jin, J. (2012), 'Automating digital leaf measurement: the tooth, the whole tooth, and nothing but the tooth', PloS one 7(8), e42112. DOI: 10.1371/journal.pone.0042112. 2, 11

Davis, C. C., Willis, C. G., Connolly, B., Kelly, C. and Ellison, A. M. (2015), 'Herbarium records are reliable sources of phenological change driven by climate and provide novel insights into species' phenological cueing mechanisms', American Journal of Botany 102(10), 1599-1609. DOI: 10.3732/ajb.1500237. 2

Deans, A. R., Lewis, S. E., Huala, E., Anzaldo, S. S., Ashburner, M., Balhoff, J. P., Blackburn, D. C., Blake, J. A., Burleigh, J. G., Chanet, B. et al. (2015), 'Finding our way through phenotypes', PLoS Biology 13(1). DOI: 10.1371/journal.pbio.1002033. 16 
Dellinger, A. S., Artuso, S., Pamperl, S., Michelangeli, F. A., Penneys, D. S., Fernández-Fernández, D. M., Alvear, M., Almeda, F., Armbruster, W. S., Staeder, Y. et al. (2019), 'Modularity increases rate of floral evolution and adaptive success for functionally specialized pollination systems', Communications Biology 2(1), 1-11. DOI: 10.1038/s42003-019-0697-7. 12

Egan, A. N. (2015), 'Species delimitation and recognition in the Pediomelum megalanthum complex (Fabaceae) via multivariate morphometrics', PhytoKeys 44, 65. DOI: 10.3897/phytokeys.44.8750. 4

Enders, C. K. (2008), 'A note on the use of missing auxiliary variables in full information maximum likelihood-based structural equation models', Structural Equation Modeling: A Multidisciplinary Journal 15(3), 434-448. DOI: 10.1080/10705510802154307. 14

Falkingham, P. L. (2012), 'Acquisition of high resolution three-dimensional models using free, opensource, photogrammetric software', Palaeontologia electronica 15(1), 15. DOI: 10.26879/264. 2

Favret, C. and Sieracki, J. M. (2016), 'Machine vision automated species identification scaled towards production levels', Systematic Entomology 41(1), 133-143. DOI: 10.1111/syen.12146. 2

Frane, J. W. (1976), 'Some simple procedures for handling missing data in multivariate analysis', Psychometrika 41(3), 409-415. DOI: 10.1007/bf02293565. 14

Gallaher, T. J., Adams, D. C., Attigala, L., Burke, S. V., Craine, J. M., Duvall, M. R., Klahs, P. C., Sherratt, E., Wysocki, W. P. and Clark, L. G. (2019), 'Leaf shape and size track habitat transitions across forestgrassland boundaries in the grass family (Poaceae)', Evolution 73(5), 927-946. DOI: 10.1111/evo.13722. 12

Gehan, M. A., Fahlgren, N., Abbasi, A., Berry, J. C., Callen, S. T., Chavez, L., Doust, A. N., Feldman, M. J., Gilbert, K. B., Hodge, J. G., Hoyer, J. S., Lin, A., Liu, S., Lizárraga, C., Lorence, A., Miller, M., Platon, E., Tessman, M. and Sax, T. (2017), 'PlantCV v2: Image analysis software for high-throughput plant phenotyping', PeerJ 5, e4088. DOI: 10.7717/peerj.4088. 2

Gehan, M. A. and Kellogg, E. A. (2017), 'High-throughput phenotyping', American Journal of Botany 104(4), 505-508. DOI: 10.3732/ajb.1700044. 15

Guillerme, T. and Cooper, N. (2018), 'Time for a rethink: time sub-sampling methods in disparitythrough-time analyses', Palaeontology 61 (4), 481-493. DOI: 10.1111/pala.12364. 12

Hemel, J. B., van der Voet, H., Hindriks, F. R. and van der Slik, W. (1987), 'Stepwise deletion: a technique for missing-data handling in multivariate analysis', Analytica Chimica Acta 193, 255-268. DOI: 10.1016/s0003-2670(00)86157-7. 14

Hereld, M. and Ferrier, N. (2019), 'LightningBug ONE: An experiment in high-throughput digitization of pinned insects', Biodiversity Information Science and Standards 3, e37228. DOI: biss.3.37228. 15 
Hereld, M., Ferrier, N. J., Agarwal, N. and Sierwald, P. (2017), Designing a high-throughput pipeline for digitizing pinned insects, in '2017 IEEE 13th International Conference on e-Science (e-Science)', IEEE, pp. 542-550. DOI: 10.1109/eScience.2017.88. 15

Horton, N. J. and Kleinman, K. P. (2007), 'Much ado about nothing: A comparison of missing data methods and software to fit incomplete data regression models', The American Statistician 61(1), 79-90. DOI: 10.1198/000313007X172556. 14

Hoyo, Y. and Tsuyuzaki, S. (2013), 'Characteristics of leaf shapes among two parental Drosera species and a hybrid examined by canonical discriminant analysis and a hierarchical Bayesian model', American Journal of Botany 100(5), 817-823. DOI: 10.3732/ajb.1200510. 9

Keklikoglou, K., Faulwetter, S., Chatzinikolaou, E., Wils, P., Brecko, J., Kvaček, J., Metscher, B. and Arvanitidis, C. (2019), 'Micro-computed tomography for natural history specimens: a handbook of best practice protocols', European Journal of Taxonomy (522). DOI: doi.org/10.5852/ejt.2019.522. 2

Klingenberg, C. P. and Gidaszewski, N. A. (2010), 'Testing and quantifying phylogenetic signals and homoplasy in morphometric data', Systematic Biology 59(3), 245-261. DOI: 10.1093/sysbio/syp106. 12

König, C., Weigelt, P., Schrader, J., Taylor, A., Kattge, J. and Kreft, H. (2019), 'Biodiversity data integration - The significance of data resolution and domain', PLoS biology 17(3), e3000183. DOI: 10.3897/biss.3.37381. 14

Kučera, J., Lihova, J. and Marhold, K. (2006), 'Taxonomy and phylogeography of Cardamine impatiens and C. pectinata (Brassicaceae)', Botanical Journal of the Linnean Society 152(2), 169-195. DOI: 10.1111/j.1095-8339.2006.00559.x. 4

Laing, A. M., Doyle, S., Gold, M. E. L., Nesbitt, S. J., O’Leary, M. A., Turner, A. H., Wilberg, E. W. and Poole, K. E. (2018), 'Giant taxon-character matrices: the future of morphological systematics', Cladistics 34(3), 333-335. DOI: 10.1111/cla.12197. 2

Law, W. and Salick, J. (2005), 'Human-induced dwarfing of Himalayan snow lotus, Saussurea laniceps (Asteraceae)', Proceedings of the National Academy of Sciences 102(29), 10218-10220. DOI: 10.1073/pnas.0502931102. 2

Le Bras, G., Pignal, M., Jeanson, M. L., Muller, S., Aupic, C., Carré, B., Flament, G., Gaudeul, M., Gonçalves, C., Invernón, V. R., Jabbour, F., Lerat, E., Lowry, P. P., Offroy, B., Pimparé, E. P., Poncy, O., Rouhan, G. and Haevermans, T. (2017), 'The French Muséum national d'histoire naturelle vascular plant herbarium collection dataset', Scientific data 4, 170016. DOI: 10.1038/sdata.2017.16. 2, 16

Little, R. J. and Rubin, D. B. (2019), Statistical analysis with missing data, Vol. 793, John Wiley \& Sons. DOI: 10.1002/9781119482260. 14 
MacLeod, N. (2007), Automated taxon identification in systematics: theory, approaches and applications, CRC Press. DOI: 10.1201/9781420008074. 15

MacLeod, N. and Steart, D. (2015), 'Automated leaf physiognomic character identification from digital images', Paleobiology 41(4), 528-553. DOI: 10.1017/pab.2015.13. 15

Mantle, B. L., La Salle, J. and Fisher, N. (2012), 'Whole-drawer imaging for digital management and curation of a large entomological collection', ZooKeys (209), 147. DOI: 10.3897/zookeys.209.3169. 2

Marhold, K. (1992), 'A multivariate morphometric study of the Cardamine amara group (Cruciferae) in the Carpathian and Sudeten mountains', Botanical Journal of the Linnean Society 110(2), 121-135. DOI: 10.1111/j.1095-8339.1992.tb00286.x. 4

Martineau, M., Conte, D., Raveaux, R., Arnault, I., Munier, D. and Venturini, G. (2017), 'A survey on imagebased insect classification', Pattern Recognition 65, 273-284. DOI: 10.1016/j.patcog.2016.12.020. 2

Melo, D., Garcia, G., Hubbe, A., Assis, A. P. and Marroig, G. (2015), 'EvolQG - An R package for evolutionary quantitative genetics', F1000Research 4. DOI: 10.12688/f1000research.7082.3. 6, 11

Musil, C. M., Warner, C. B., Yobas, P. K. and Jones, S. L. (2002), 'A comparison of imputation techniques for handling missing data', Western Journal of Nursing Research 24(7), 815-829. DOI: 10.1177/019394502762477004. 14

O’Leary, M. A., Bloch, J. I., Flynn, J. J., Gaudin, T. J., Giallombardo, A., Giannini, N. P., Goldberg, S. L., Kraatz, B. P., Luo, Z.-X., Meng, J., Ni, X., Novacek, M., Perini, F., Randall, Z., Rougier, G., Sargis, E., Silcox, M., Simmons, N., Spaulding, M., Velazco, P., Weksler, M., Wible, J. and Cirranello, A. (2013), 'The placental mammal ancestor and the post-K-Pg radiation of placentals', Science 339(6120), 662-667. DOI: $10.7934 / \mathrm{p} 773.2$

O’Leary, M. A., Alphonse, K., Mariangeles, A. H., Cavaliere, D., Cirranello, A., Dietterich, T. G., Julius, M., Kaufman, S., Law, E., Passarotti, M., Reft, A., Robalino, J., Simmons, N. B., Smith, S. Y., Stevenson, D. W., Theriot, E., Velazco, P. M., Walls, R. L., Yu, M. and Daly, M. (2018), 'Crowds replicate performance of scientific experts scoring phylogenetic matrices of phenotypes', Systematic Biology 67(1), 49-60. DOI: 10.1093/sysbio/syx052. 15

O'Leary, M. A. and Kaufman, S. G. (2011), 'MorphoBank: phylophenomics in the "cloud”', Cladistics 27(5), 529-537. DOI: 10.1111/j.1096-0031.2011.00355.x. 15

Pearson, K. (1920), 'Notes on the history of correlation', Biometrika 13(1), 25-45. DOI: 10.1093/biomet/13.1.25. 5

Poulsen, A. D. and Nordal, I. (2005), 'A phenetic analysis and revision of Guineo-Congolean rain forest taxa of Chlorophytum (Anthericaceae)', Botanical Journal of the Linnean Society 148(1), 1-20. DOI: 10.1111/j.1095-8339.2005.00386.x. 4 
Price, B. W., Dupont, S., Allan, E. L., Blagoderov, V., Butcher, A. J., Durrant, J., Holtzhausen, P., Kokkini, P., Livermore, L., Hardy, H. et al. (2018), 'ALICE: Angled Label Image Capture and Extraction for high throughput insect specimen digitisation'. DOI: 10.31219/osf.io/s2p73. 15

Reginato, M. and Michelangeli, F. A. (2016), 'Diversity and constraints in the floral morphological evolution of Leandra s. str. (Melastomataceae)', Annals of Botany 118(3), 445-458. DOI: 10.1093/aob/mcw116. 15

Remagnino, P., Mayo, S., Wilkin, P., Cope, J. and Kirkup, D. (2016), Computational botany: methods for automated species identification, Springer. DOI: $10.18520 / \mathrm{cs} / \mathrm{v1} 15 / \mathrm{i} 2 / 349-350.2,15$

Rose, J. P. and Freudenstein, J. V. (2014), 'Cryptic and overlooked: species delimitation in the mycoheterotrophic Monotropsis (Ericaceae: Monotropoideae)', Systematic Botany 39(2), 578-593. DOI: 10.1600/036364414x680762. 4

Roweis, S. T. (1998), EM algorithms for PCA and SPCA, in M. I. Jordan, M. J. Kearns and S. A. Solla, eds, 'Advances in neural information processing systems', pp. 626-632. 5, 14

Rubin, D. B. (1976), 'Inference and missing data', Biometrika 63(3), 581-592. DOI: 10.1093/biomet/63.3.581. 14

Saunders, J. A., Morrow-Howell, N., Spitznagel, E., Doré, P., Proctor, E. K. and Pescarino, R. (2006), 'Imputing missing data: A comparison of methods for social work researchers', Social Work Research 30(1), 19-31. DOI: 10.1093/swr/30.1.19. 14

Schmidt, S., Balke, M. and Lafogler, S. (2012), 'DScan - a high-performance digital scanning system for entomological collections', ZooKeys (209), 183. DOI: 10.3897/zookeys.209.3115. 2

Simon, M. N. and Marroig, G. (2015), 'Landmark precision and reliability and accuracy of linear distances estimated by using 3D computed micro-tomography and the open-source TINA Manual Landmarking Tool software', Frontiers in Zoology 12(1), 12. DOI: 10.1186/s12983-015-0101-5. 9

Slovák, M., Kučera, J., Marhold, K. and Zozomová-Lihová, J. (2012), 'The morphological and genetic variation in the polymorphic species Picris hieracioides (Compositae, Lactuceae) in Europe strongly contrasts with traditional taxonomical concepts', Systematic Botany 37(1), 258-278. DOI: 10.1600/036364412x616828. 4

Smith, S. D. and Kriebel, R. (2018), 'Convergent evolution of floral shape tied to pollinator shifts in Iochrominae (Solanaceae)', Evolution 72(3), 688-697. DOI: 10.1111/evo.13416. 15

Soltis, P. S. (2017), 'Digitization of herbaria enables novel research', American Journal of Botany 104(9), 1281-1284. DOI: 10.3732/ajb.1700281. 2, 16 
Stacklies, W., Redestig, H., Scholz, M., Walther, D. and Selbig, J. (2007), 'pcamethods - a bioconductor package providing pca methods for incomplete data', Bioinformatics 23, 1164-1167. DOI: 10.1093/bioinformatics/btm069. 5

Streiner, D. L. and Norman, G. R. (2006), “Precision” and "accuracy”: two terms that are neither', Journal of Clinical Epidemiology 59(4), 327-330. DOI: 10.1016/j.jclinepi.2005.09.005. 3

Ströbel, B., Schmelzle, S., Blüthgen, N. and Heethoff, M. (2018), 'An automated device for the digitization and 3D modelling of insects, combining extended-depth-of-field and all-side multi-view imaging', ZooKeys (759), 1. 15

Suarez, A. V. and Tsutsui, N. D. (2004), 'The value of museum collections for research and society', AIBS Bulletin 54(1), 66-74. DOI: 10.1641/0006-3568(2004)054[0066:TVOMCF]2.0.CO;2. 2

Sweeney, P. W., Starly, B., Morris, P. J., Xu, Y., Jones, A., Radhakrishnan, S., Grassa, C. J. and Davis, C. C. (2018), 'Large-scale digitization of herbarium specimens: Development and usage of an automated, high-throughput conveyor system', Taxon 67(1), 165-178. DOI: 10.12705/671.10. 11, 14

Takano, A., Horiuchi, Y., Fujimoto, Y., Aoki, K., Mitsuhashi, H. and Takahashi, A. (2019), 'Simple but longlasting: A specimen imaging method applicable for small-and medium-sized herbaria', PhytoKeys 118, 1-14. DOI: 10.3897/phytokeys.118.29434. 11, 14

Tegelberg, R., Mononen, T. and Saarenmaa, H. (2014), 'High-performance digitization of natural history collections: Automated imaging lines for herbarium and insect specimens', Taxon 63(6), 1307-1313. DOI: $10.12705 / 636.13 .15$

Thiers, B. (2020, continuously updated), 'Index Herbariorum: A global directory of public herbaria and associated staff. New York Botanical Garden’s Virtual Herbarium', http://sweetgum. nybg. org/ih . 3

Trovó, M., Sano, P. and Winkworth, R. (2008), 'Morphology and environment: geographic distribution, ecological disjunction, and morphological variation in Actinocephalus polyanthus (Bong.) Sano (Eriocaulaceae)', Feddes Repertorium 119(7-8), 634-643. DOI: 10.1002/fedr.200811189. 4, 11

Tulig, M., Tarnowsky, N., Bevans, M., Kirchgessner, A. and Thiers, B. M. (2012), 'Increasing the efficiency of digitization workflows for herbarium specimens', ZooKeys 209, 103-113. DOI: 10.3897/zookeys.209.3125. 11, 14

Unger, J., Merhof, D. and Renner, S. (2016), 'Computer vision applied to herbarium specimens of German trees: testing the future utility of the millions of herbarium specimen images for automated identification', BMC Evolutionary Biology 16(1), 248. DOI: 10.1186/s12862-016-0827-5. 15

Vasconcelos, T. N., Chartier, M., Prenner, G., Martins, A. C., Schönenberger, J., Wingler, A. and Lucas, E. (2018), 'Floral uniformity through evolutionary time in a species-rich tree lineage', New Phytologist . DOI: $10.1111 /$ nph.15453. 12 
Wang, J., Ji, L., Liang, A. and Yuan, D. (2012), 'The identification of butterfly families using content-based image retrieval', Biosystems engineering 111(1), 24-32. DOI: 10.1016/j.biosystemseng.2011.10.003. 2, 15

Wang, J., Lin, C., Ji, L. and Liang, A. (2012), 'A new automatic identification system of insect images at the order level', Knowledge-Based Systems 33, 102-110. DOI: 10.1016/j.knosys.2012.03.014. 2, 15

Wiens, J. J. (2003), 'Missing data, incomplete taxa, and phylogenetic accuracy', Systematic Biology 52(4), 528-538. DOI: 10.1080/10635150390218330. 11

Wilcoxon, F. (1945), 'Individual comparisons by ranking methods', Biometrics Bulletin 1(6), 80-83. DOI: 10.2307/3001968. 4

Willis, C. G., Ellwood, E. R., Primack, R. B., Davis, C. C., Pearson, K. D., Gallinat, A. S., Yost, J. M., Nelson, G., Mazer, S. J., Rossington, N. L. et al. (2017), 'Old plants, new tricks: Phenological research using herbarium specimens', Trends in Ecology \& Evolution 32(7), 531-546. DOI: 10.1016/j.tree.2017.03.015. 16

Yang, K. and Shahabi, C. (2004), A PCA-based similarity measure for multivariate time series, in 'Proceedings of the 2nd ACM International Workshop on Multimedia Databases', pp. 65-74. DOI: 10.1145/1032604.1032616. 6, 11

Yezerinac, S. M., Lougheed, S. C. and Handford, P. (1992), 'Measurement error and morphometric studies: statistical power and observer experience', Systematic Biology 41 (4), 471-482. DOI: 10.2307/2992588. $9,11,12$

Younis, S., Weiland, C., Hoehndorf, R., Dressler, S., Hickler, T., Seeger, B. and Schmidt, M. (2018), 'Taxon and trait recognition from digitized herbarium specimens using deep convolutional neural networks', Botany Letters 165(3-4), 377-383. DOI: 10.1080/23818107.2018.1446357. 11

Zaragüeta-Bagils, R. and Bourdon, E. (2007), 'Three-item analysis: Hierarchical representation and treatment of missing and inapplicable data', Comptes Rendus Palevol 6(6-7), 527-534. DOI: 10.1016/j.crpv.2007.09.013. 12

Zhou, N., Siegel, Z. D., Zarecor, S., Lee, N., Campbell, D. A., Andorf, C. M., Nettleton, D., LawrenceDill, C. J., Ganapathysubramanian, B., Kelly, J. W. et al. (2018), 'Crowdsourcing image analysis for plant phenomics to generate ground truth data for machine learning', PLoS Computational Biology 14(7), e1006337. DOI: 10.1371/journal.pcbi.1006337. 15 